\title{
Toxoplasma gondii Hsp90: potential roles in essential cellular processes of the parasite
}

\author{
SERGIO O. ANGEL ${ }^{1}$, MARIA J. FIGUERAS ${ }^{1}$, MARIA L. ALOMAR $^{1}$, \\ PABLO C. ECHEVERRIA ${ }^{2}$ and BIN DENG ${ }^{3}$ \\ ${ }^{1}$ Laboratorio de Parasitología Molecular, IIB-INTECH, CONICET-UNSAM, Av. Intendente Marino Km. 8·2, \\ C.C 164, (B7130IIWA), Chascomús, Prov. Buenos Aires, Argentina \\ ${ }^{2}$ Département de Biologie Cellulaire Université de Genève Sciences III, Geneva, Switzerland \\ ${ }^{3}$ Biology Department, Vermont Genetics Network Proteomics Facility, The University of Vermont, 337 marsh Life Science \\ Building, 109 Carrigan Drive, Burlington, VT 05405, USA
}

(Received 15 October 2013; revised 30 December 2013; accepted 4 Fanuary 2014; first published online 21 February 2014)

SUMMAR Y

Hsp90 is a widely distributed and highly conserved molecular chaperone that is ubiquitously expressed throughout nature, being one of the most abundant proteins within non-stressed cells. This chaperone is up-regulated following stressful events and has been involved in many cellular processes. In Toxoplasma gondii, Hsp90 could be linked with many essential processes of the parasite such as host cell invasion, replication and tachyzoite-bradyzoite interconversion. A Protein-Protein Interaction (PPI) network approach of TgHsp90 has allowed inferring how these processes may be altered. In addition, data mining of $T$. gondii phosphoproteome and acetylome has allowed the generation of the phosphorylation and acetylation map of TgHsp90. This review focuses on the potential roles of $\operatorname{TgHsp} 90$ in parasite biology and the analysis of experimental data in comparison with its counterparts in yeast and humans.

Key words: Toxoplasma gondii, Hsp90, cell cycle, post-translational modification, development.

TOXOPLASMA GONDII AND TOXOPLASMOSIS

Toxoplasma gondii is an obligate intracellular protozoan parasite that belongs to the phylum Apicomplexa (Dubey et al. 1970). This phylum includes important protozoan parasites such as Plasmodium spp., Cryptosporidium spp. and Eimeria spp. among others. Toxoplasma gondii is the causative agent of toxoplasmosis, a disease spread worldwide with about 500 million to a billion people chronically infected (Tenter et al. 2000). Toxoplasma gondii is able to infect almost any nucleated cell of warmblooded animals, including mammals and birds (Dubey et al. 1970).

Toxoplasma gondii may undergo both cycles of sexual reproduction within the definitive host (any member of the cat family) and cycles of asexual reproduction within any warm-blooded animal, including humans (Dubey et al. 1970). Inside intermediate hosts, $T$. gondii presents two stages of asexual development: the rapidly growing tachyzoites, responsible for the acute illness, and the slowly dividing bradyzoites, responsible for the chronic and asymptomatic infection (Dubey et al. 1998). Tachyzoites invade many cell types and rapidly

* Corresponding author. Laboratorio de Parasitología Molecular, IIB-INTECH, CONICET-UNSAM, Av. Intendente Marino Km. 8.2, C.C 164, (B7130IIWA), Chascomús, Prov. Buenos Aires, Argentina. E-mail: sangel@intech.gov.ar multiply by repeated endodyogeny inside a parasitophorous vacuole (PV) until disruption of the host cell and the subsequent invasion of nearby cells (Dubey et al. 1998). In response to stress factors, usually in reaction to the host immune system, tachyzoites can convert to bradyzoites, forming tissue cysts which may persist as latent cysts for the rest of the host's life (Dubey et al. 1998). Within tissue cysts, bradyzoites replicate slowly by endodyogeny (Dubey et al. 1970). Cysts can be ingested by a definitive host, in which case the parasite undergoes cycles of sexual reproduction (Dubey et al. 1970).

Toxoplasmosis is of medical importance because $T$. gondii can cause opportunistic diseases in immunocompromised individuals, due to reactivation of a latent infection. Toxoplasma gondii can also cause spontaneous abortion or congenital birth defects in newborns if the mother is primo-infected during pregnancy (Carlier et al. 2012). The active form of the parasite can cause encephalitis and neurological diseases, and can also affect heart, liver, inner ears and eyes (chorioretinitis). Recently, toxoplasmosis has been linked with schizophrenia and other neurological disorders and behaviour alterations (Miman et al. 2010; Park et al. 2012; Halonen and Weiss, 2013). Despite the existence of effective drug regimens, in some cases, the therapy is not well tolerated by the patients. In addition, there is still no effective therapy against latent infection. It is expected that specific proteins of the parasite, 
which play important or essential roles in $T$. gondii pathogenesis, allow the generation of novel therapies that can overcome some of the afore-mentioned situations. In this regard, the chaperone heat shock protein 90 (Hsp90) has arisen as an interesting drug target against acute and latent toxoplasmosis (Shonhai et al. 2011; Angel et al. 2013; Roy et al. 2012; Rochani et al. 2013).

\section{HSP 90: STRUCTURE AND COMPLEXES}

Hsp90 is a widely distributed and highly conserved molecular chaperone that is ubiquitously expressed throughout nature, being one of the most abundant proteins within non-stressed cells (Picard, 2002; Pratt and Toft, 2003; Tsutsumi et al. 2009). Rather than binding any unfolded protein, Hsp90 binds more than 200 client proteins which require it for the correct activation of key cellular processes such as control of genetic expression, cell cycle progression, apoptosis, cancer, stress response, plant immunity, development and even evolutionary processes (McClellan et al. 2007; Bogumil and Dagan, 2012; Jackson, 2013). Hsp90 is an ATP-dependent chaperone whose ATPase activity is regulated by the binding of co-chaperones and substrates (Prodromou and Pearl, 2003). This binding can be specifically inhibited by ansamycin-benzoquinone antibiotics (Geldanamycin-GA- and its derivatives) and Radicicol. These antibiotics bind specifically to the Hsp90 ATP binding pocket with higher affinity than ATP itself (Stebbins et al. 1997). GA specifically inhibits the ATPase activity of Hsp90 as a result of its competition with ATP for binding to the N-terminal domain nucleotide binding pocket (Prodromou et al. 1997). This mechanism interferes with the maturation process of client proteins, facilitating their ubiquitin-mediated proteasomal degradation (Pearl et al. 2008). This can be used as an important tool for the analysis of Hsp90 functions and the identification of its client proteins.

The Hsp90 structure consists of three highly conserved domains: an N-terminal domain (ATP binding site, GA binding site, p23, Aha 1 and Cdc37 co-chaperones binding site), a middle domain (catalytic loop required for ATPase activity, Aha1 co-chaperone binding site) and a C-terminal domain (dimerization interface) (Retzlaff et al. 2010; Jackson, 2013). In addition, it has a charged linker region and the MEEVD motif (which binds TPR-containing cochaperones). Several co-chaperones (e.g. p50/Cdc37, p60Hop/Sti1, p23, Aha1) modulate Hsp90 ATPase activity, thereby altering the cycling rate of the chaperone machine (Li et al. 2012). The composition of co-chaperone complexes varies according to the client proteins and the pathway in which the chaperone is involved ( $\mathrm{Li}$ and Buchner, 2013). In higher eukaryotes, it is possible to define at least four Hsp90 cycles: (i) the Hsp70/Hsp90 cycle or
Hsp90-heterocomplex for steroid hormone receptors; (ii) the Hsp70/Hsp90 cycle or Hsp90-heterocomplex for protein kinases (which involves cdc37 protein); (iii) a cycle that involves SGT1 cochaperones, which in plants plays a role in immune sensors, but it is also associated with the kinetochore assembly pathway; and (iv) Hsp90 complexes associated with RNA processing ( $\mathrm{Li}$ and Buchner, 2013). Hsp90-heterocomplexes consist of sequential complexes formed by Hsp90 and different cochaperones. These are assembled during protein client maturation forming dynamic multi-chaperone complexes. They could be defined as early, intermediate and late Hsp70/Hsp90 cycles ( $\mathrm{Li}$ and Buchner, 2013).

TOXOPLASMA GONDII HSP90 AND ITS CYCLES

Toxoplasma gondii presents two of the abovementioned Hsp90 cycles (Fig. 1). In the case of the T. gondii Hsp90 (TgHsp90)-heterocomplex, $T$. gondii seems to have only one of these pathways because the cdc 37 protein has not been detected. In our laboratory, we have identified all the chaperones and co-chaperones associated with TgHsp90heterocomplex (Echeverria et al. 2010; Figueras et al. 2012, unpublished).We have also detected an early/intermediate and a late TgHsp90 complex similar to those found in higher eukaryotes (Fig. 1) (Echeverria et al. 2010). The early/intermediate TgHsp90 complex is composed at least by HIPHsp70-TgHsp90 whereas the late complex is composed by p23-TgHsp90. In addition, p23 and TgHsp90 was shown to be cytosolic during the tachyzoite stage and both cytosolic and nuclear during the bradyzoite stage, whereas HIP, Sis1 and HOP was shown to be cytosolic during both stages of asexual reproduction in vitro (Echeverria et al. 2005; Ueno et al. 2011; Figueras et al. 2012, unpublished). These data indicate that both p23 and TgHsp90 could be engaged to a multitude of large protein complexes as has been observed in higher eukaryotes (Picard et al. 2006).

The Hsp90 protein also participates in the complex involved in the early kinetochore assembly pathway, as observed in yeast (Fig. 1) (Makhnevych and Houry, 2012). Mining the T. gondii database (www.toxodb.org) it was possible to reconstruct the kinetochore assembly pathway and two putative SGT1 co-chaperones could be detected (Fig. 1). SGT1 proteins belong to a group of proteins that have different domains, including the CS (CHORD [cysteine and histidine-rich domain]-Sgt1 domain (sometimes called p23-like or alpha crystallin domain) and the SGS domain (Sgt1-specific domain) at the carboxy terminus. In addition, SGT1 proteins have a TPR domain that links Skp1 to the core of the Hsp90 chaperone (Catlett and Kaplan, 2006). Toxoplasma gondii has two putative sequences 
A

\section{T. gondii Hsp90-heterocomplex}
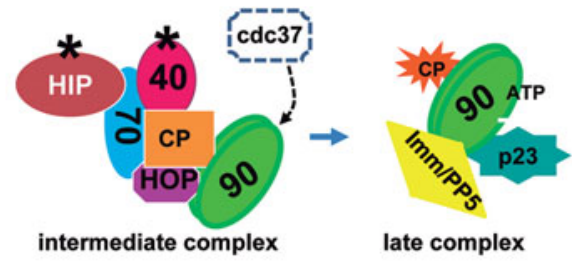

B

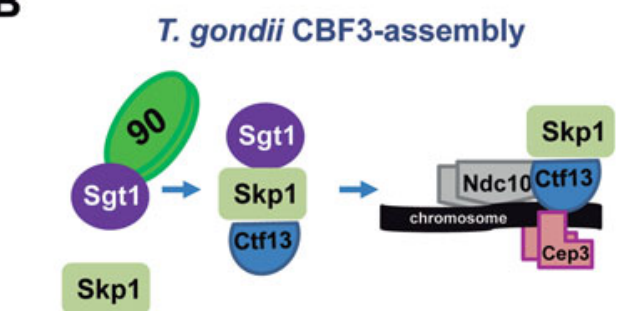

C

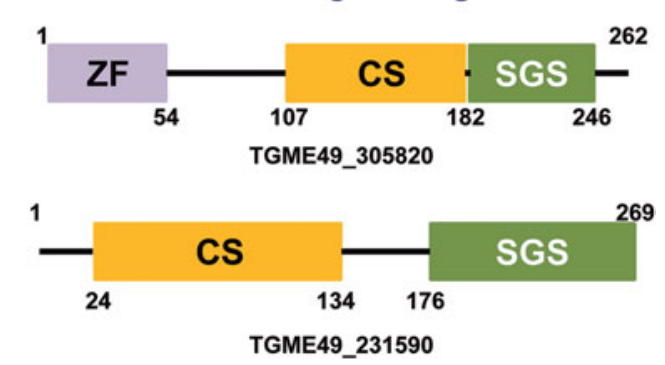

Fig. 1. Hsp90 cycles present in T. gondii. (A) Hsp90/ Hsp70 cycle or TgHsp90-heterocomplex for transcription factors/protein kinases. The compositions of the early/ intermediate complex and of the late complex are shown on the left and right hand, respectively. The cochaperone cdc37 binds to the Hsp90/Hsp70 cycle associated to kinases in higher eukaryotes. Since it was not identified in the $T$. gondii database, it is indicated with a dashed line. (B) The T. gondii CBF3-assembly complex. (C) The sequence domain organization of the two putative SGT1s identified in the $T$. gondii database. 90: TgHsp90; 70: Hsp70; HIP: Hsp70 interacting protein; HOP: Hsp70/Hsp90 organizing protein; 40: Hsp40; CP: Client Protein; Imm/PP5: Immunophilin or PP5; P23: p23; Sgt1: suppressor of G2 allele of SKP1, S-phase kinase-associated protein 1; Ctf13: Chromosome transmission fidelity protein 13 ; Ndc10: is also named $\mathrm{CBF} 2$ for centromere binding factor 2; Cep3: is also named $\mathrm{CBF} 3 \mathrm{~b}$ for centromere binding factor $3 \mathrm{~b}$.

encoding SGT1, identified as TGME49_305820 and TGME49_231590 (Fig. 1C). None of them has a TPR domain but TGME49_305820 has a zinc finger domain at its amino terminus, not observed in other SGT1 proteins.

In addition to these Hsp90 cycles, this chaperone has been found to be associated with RNA processing in yeast, involving Pih1, Tah1 and Rvb1 and Rvb2 (Li and Buchner, 2013). Mining the T. gondii database has not retrieved any orthologue of Pih1 and Tah1 (Suvorova et al. 2013). However, the data obtained from a combined analysis of co-immunoprecipitation and mass spectrometry assays using anti-TgHsp90 antibody and orthologous partners of TgHsp90 from a Plasmodium falciparum ProteinProtein Interaction (PPI) network database (see Table S1) allowed the retrieval of several putative TgHsp90 interactors involved in RNA processing. These data suggest that TgHsp90 would have an important role in this process and thus could become a very interesting field for future studies.

POST-TRANSLATIONAL MODIFICATIONS

Hsp90 function can be altered by different posttranslational modifications (PTMs) such as phosphorylation, acetylation, S-nitrosylation, oxidation and ubiquitination (Mollapour and Neckers, 2012). Recently, the phosphoproteome and acetylome of T. gondii have been determined (Treeck et al. 2011; Jeffers and Sullivan, 2012; Xue et al. 2013). These data have been incorporated in the www.toxodb.org database allowing us to generate a PTM map of TgHsp90 (Fig. 2, Table 1). Nonetheless these modifications should be further confirmed by other methods.

Toxoplasma gondii Hsp90 would be phosphorylated at several sites along its sequence (Table 1); most of them (except S464) are also modified in human or yeast Hsp90 orthologues (Mollapour et al. 2010a; Mollapour and Neckers, 2012; Soroka et al. 2012). Interestingly, human and yeast Hsp90s have other phosphorylated sites of which PTMs was not detected in TgHsp90 despite the conserved residues in the parasite chaperone (Table 1). Although the role of Hsp90 phosphorylation in $T$. gondii has not yet been studied, it is known that phosphorylation has important implications in the regulation of the Hsp90 structure and function in other organisms. Mutation of S485 in yeast Hsp90 is lethal, altering the dynamics of the conformational changes (Soroka et al. 2012). In some cases, phosphorylation of Hsp90 reduces the avidity for its client protein. The lack of phosphorylation of S226/S255 and $\mathrm{S} 231$ on the linker region of HuHsp90 $\beta$ and HuHsp90 $\alpha$, respectively, increases the binding affinity of the chaperones for the transcription factor arylhydrocarbon receptor (Ogiso et al. 2004). The treatment of $v$-src-transfected cells with okadaic acid, a serine/threonine phosphatase inhibitor, destabilizes the Hsp90-pp60 $60^{\mathrm{v}-\mathrm{src}}$ chaperone complex (Mimnaugh et al. 1995). Mutation of T22 on yeast Hsp90 (T23 in TgHsp90, Table 1) results in the accumulation of less glucocorticoid receptor (GR) protein than wild-type cells and reduces GR and pp $60^{\mathrm{v}-\mathrm{src}}$ activities in a yeast model (Nathan and Lindquist, 1995). More recently, it has been observed that phosphorylation of T22 in yeast Hsp90 affects its ATPase activity and also its function on 


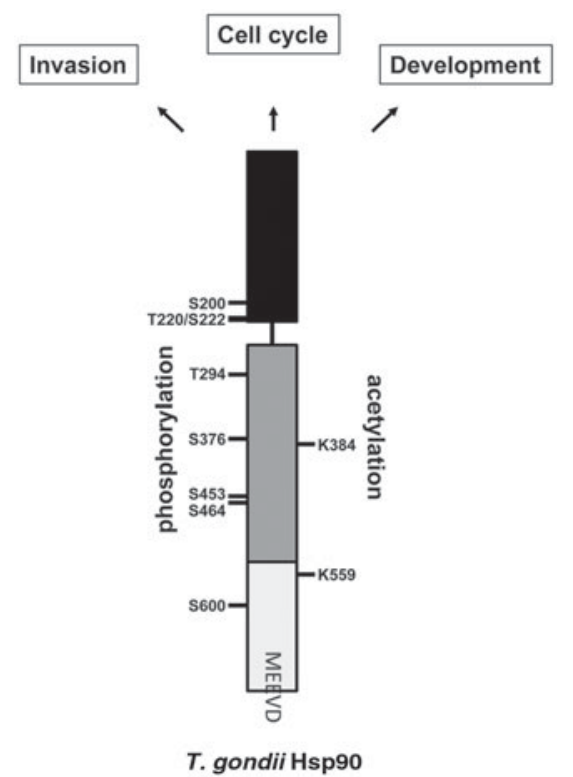

Fig. 2. Schematic representation of TgHsp90. In black: The N-terminal domain, in grey: the central domain and in light grey: the $\mathrm{C}$-terminal domain. Highlighted are the amino acids MEEVD and the residues that are identified to have received post-translational modifications (PTMs). One letter code was used to indicate the corresponding amino acids. The panel also shows the biological processes in which TgHsp90, more likely through the TgHsp90-heterocomplex, is involved: modulation of invasion, cell cycle and differentiation.

several client proteins, either kinases or non-kinases, affecting the interaction between the Hsp90 protein and co-chaperones (Mollapour et al. 2011a,b). By contrast, indirect analysis based on the inability of PP5 phosphatase to bind the Hsp90-heme-regulated inhibitor of protein synthesis (HRI) has shown that chaperone phosphorylation enhances HRI activity (Shao et al. 2002). Phosphorylation of tyrosine 197 in human Hsp90a (Y186 in TgHsp90, Table 1) dissociates cdc37 from Hsp90 (Xu et al. 2012). This study also showed that mutation of Y627 (Y604 in $T$. gondii, Table 1) favours the release of client Cdk4 and co-chaperones (AHA1 and PP5). Wang et al. (2009) have shown that phosphorylation of T90 by protein kinase A (PKA) stimulates the cellular secretion of human Hsp90 $\alpha$.

Phosphorylation of Hsp90 is mediated by several kinases such as DNA-dependent protein kinase (DNA-PK), casein kinase II (CK2), PKA, c-Src and Wee1 (Mollapour et al. 2010b; Mollapour and Neckers 2012). The phosphorylation status is also regulated by PP5 and Ppt1 phosphatases (Mollapour et al. 2010b). Their T. gondii putative counterparts are: CK2 (TGME49_272400); PKA (TGME49_ 242070); DNA-PK (TGME49_266000); NEK [Wee1] (TGME49_319700); c-Src (TGME49_272475); Ppt1 (TGME49_312200); and PP5 (TGME49_ 312200). None of these kinases and phosphatases was detected as $\mathrm{TgHsp} 90 / \mathrm{p} 23$ putative interactors
(Table S1). Since PP5 is an Hsp90 co-chaperone, it is expected that TgPP5 will be pulled down with TgHsp90 in future studies.

Thus, as observed, phosphorylation of $\mathrm{Hsp} 90$ is of key importance for Hsp90 function and complex cycle. Despite the numerous putative phosphorylated sites found in $\mathrm{TgHsp} 90$, there are several phosphorylated sites in yeast and human Hsp90s with important regulatory functions, which are present in TgHsp90 but not in the phosphoproteome. It is expected that new sites of phosphorylation will be detected in TgHsp90 and that their importance and biological role would be determined.

More recently, the role of a lysine acetylation in regulating Hsp90 function has been evidenced. Human Hsp90s present several acetylable lysines along their sequence (Mollapour and Neckers, 2012). The histone acetyl-transferase (HAT) and histone deacetylase (HDAC) enzymes are involved in modulating the acetylation status of Hsp90. The acetylation of Hsp90 negatively affects the Hsp90 functions. Exposure of cancer cells to the HDAC inhibitor FK228 has been shown to block the interaction of Hsp90 with the key cell cycle regulators p53 and Raf-1 (Yu et al. 2002). In the TgHsp90 sequence, two putative acetylated lysines (K384 and K559) have been identified (Jeffers and Sullivan, 2012; Xue et al. 2013) (Fig. 2 and Table 1). These lysines are conserved in human Hsp90 and one is also acetylated (Table 1). The role of lysine K294 in human Hsp90 $\alpha$, also present in TgHsp90 (K271, Table 1), has been further studied. The replacement of this lysine by alanine (K294A) dramatically reduces the interaction of Hsp90 with the client receptor tyrosine kinase ErbB2 and the kinase p60 ${ }^{\mathrm{v}-\mathrm{Src}}$ (Scroggins et al. 2007). A similar effect has been observed with co-chaperones AHA1, FKBP52, HOP, Hsp70 and C-terminus of Hsp70interacting protein (CHIP), where the mutant has shown a reduced or undetectable level of interaction with the Hsp90 (K294A) mutant version (Scroggins et al. 2007).

The enzyme responsible for the regulation Hsp90 deacetylation is HDAC6 (class II deacetylase) whose inactivation results in $\mathrm{Hsp} 90$ hyper-acetylation, leading to the dissociation of the functional GRHsp90 and thus affecting the receptor maturation (Kovacs et al. 2005; Murphy et al. 2005). HDAC1, another class II deacetylase, has also been reported to deacetylate Hsp90 in the nucleus of human breast cancer cells (Zhou et al. 2008), and both HDAC1 and HDAC10 inhibit the productive Hsp90 chaperoning of VEGF receptor proteins (Park et al. 2008). Although HDAC6 has not been detected, other members of the HDAC class II family (such as HDAC1 and HDAC5) have been detected by mining the $T$. gondii database (Vanagas et al. 2012). However, none of them have been identified as Hsp90 interactors so far. 
Table 1. PTMs of T. gondii, yeast and human Hsp90 members

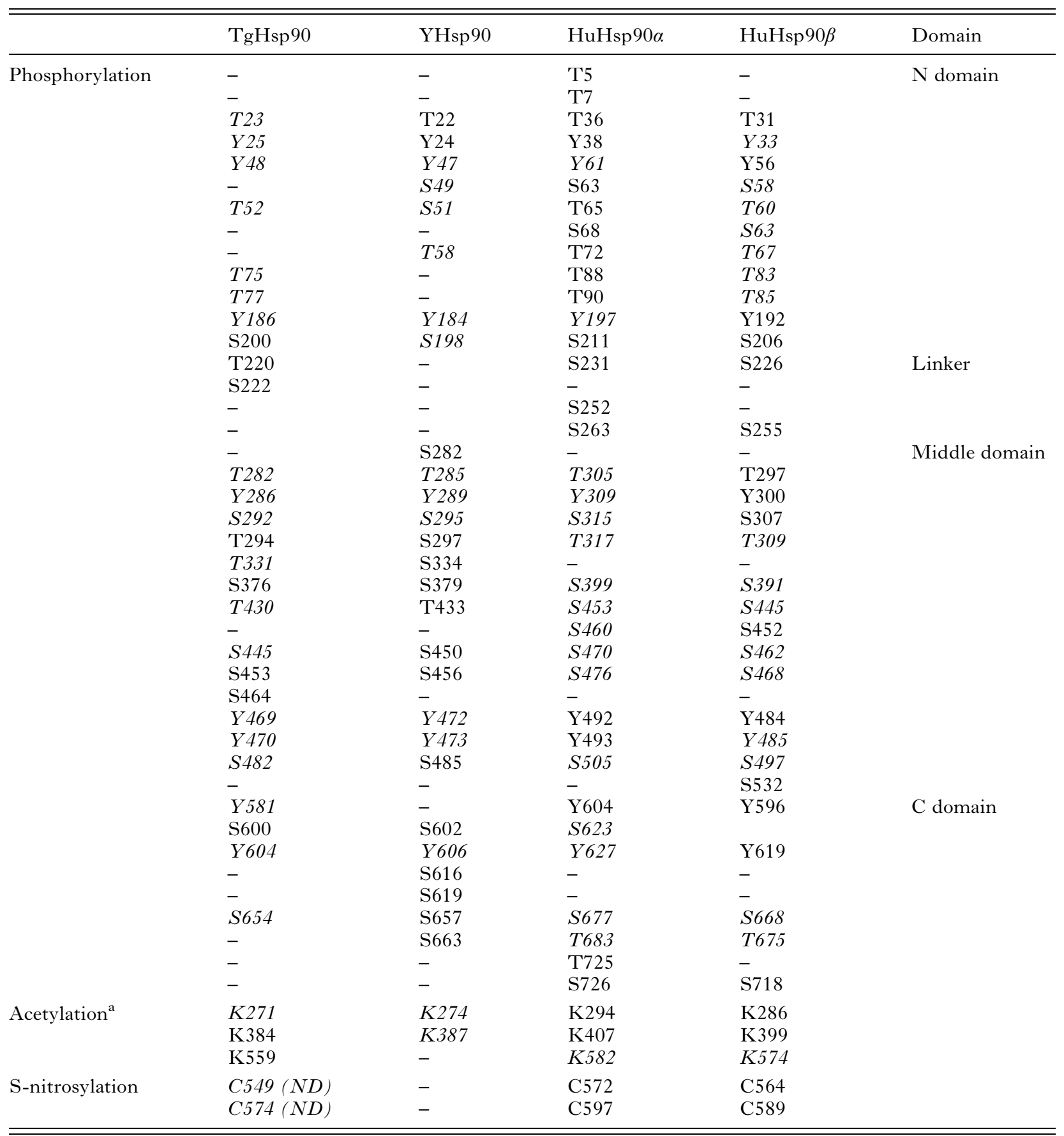

${ }^{a}$ Data only include acetylated lysines identified in T. gondii and human Hsp90. Other lysines that were detected in human Hsp90 are not included.

ND: indicates not determined; Normal font: PTMs identified; italic font: orthologue residue; -: non phosphorylated $(\mathrm{Y}, \mathrm{T}, \mathrm{S})$, acetylated $(\mathrm{K})$ or S-nitrosylated $(\mathrm{C})$ residue; Obs.: Hsp90 domains are only identified for phosphorylation section.

Acetylation of Hsp90 seems to be headed by p300/CBP (CREB-binding protein) HATs (Kovacs et al. 2005; Mollapour and Neckers, 2012). However, up to now, little is known about this issue. Although it is a recent field of study, the role of these PTMs in biological processes is gaining the interest of researchers, as phosphorylation did in the past. The exploration of acetylation/deacetylation modulation in TgHsp90 will bring on interesting findings in the future and will probably promote novel anti-Hsp90 drugs.

S-nitrosylation is a covalent attachment of a nitrogen monoxide group to the thiol side chain of a cysteine. This reversible PTM of Hsp90 is mediated by nitric oxide (NO), and has been shown to inhibit its chaperone activity (Martinez-Ruiz et al. 2005). This PTM occurs in the C-terminal domain of Hsp90 at C597, a residue also present in 
TgHsp90 ('Table 1). The S-nitrosylation has not been studied in TgHsp90 yet, being another intriguing subject for future studies.

\section{THE CELL CYCLE}

The incubation of intracellular tachyzoites with GA has a clear effect on $T$. gondii reducing the replication rate of tachyzoites (Ahn et al. 2003; Echeverria et al. 2005). Toxoplasma gondii replicates with a mechanism named endodyogeny, in which two daughter cells bud inside a mother cell (Gubbels et al. 2008). The cell cycle of highly replicative tachyzoites comprises the Gap phase G1-S-Mitosis, whereas the G2 is not detectable (Radke et al. 2001). Inside the host cell, the rate of replication is about one complete cycle every 5-9 $\mathrm{h}$ and then starts again to complete several rounds (Radke et al. 2001). Although the cell cycle resembles that of higher eukaryotes, the complete round of parasite replication has some differences: the nuclear envelope does not disappear along the parasite division (Striepen et al. 2007) and chromosomes do not present a high order level of condensation. In addition, many of the checkpoint factors that control $\mathrm{G} 1$ to $\mathrm{S}$ phase progression in higher eukaryotes are not detected in T. gondii (Gubbels et al. 2008; Behnke et al. 2010). When tachyzoites enter the bradyzoite differentiation process, the cell cycle presents a long G2-phase during early events, and then mature bradyzoites stay arrested in G0 (Radke et al. 2003). Therefore, replication and differentiation seem to be linked. Up to now, the cell signals responsible for modulating $T$. gondii replication are unknown. Moreover, it is still unknown whether there is a connection between host cell and tachyzoites in the modulation of the parasite replication process. The treatment of host cells with phosphatidylinositide 3-kinases (PI3K kinases) inhibitors such as LY294002 and wortmannin has been shown to reduce tachyzoite replication (Zhou et al. 2013). TgHsp90 PPI analysis has shown several putative kinases and phosphatases that could be involved in cell signalling (Table S1). However, the biological role of some of these proteins has not been assessed yet. We could infer that in the future some of these signalling kinases and phosphatases could be linked to Hsp90 and the cell cycle.

A clear association between Hsp90 and the cell cycle was observed early. This association involves cyclin-dependent kinase 1 CDK1 (also named cdc2 or cdc28), CDK2, CDK4, Cyclins B, D and E as well as the kinases Wee1, Myt-1, Plk1, Aurora B and survivin (Burrows et al. 2004). The inhibition of Hsp90 with XL888 blocks tumour cell progression and leads to cell cycle arrest and degradation of different client kinases such as CDK1 (cdc2/cdc28), ChK1 and Wee1 in vitro (Haarberg et al. 2013). Hsp90 is also linked to cyclin E degradation without binding to it (Bedin et al. 2009). Cyclin E regulates
CDK2 and is required to initiate DNA replication at the G1/S transition. Although most of these cell cycle associated proteins are present in the $T$. gondii database, none of them were identified as a putative TgHsp90/p23 interactor. In addition, other proteins associated with the $T$. gondii replication process could be involved as TgHsp90 client proteins (e.g. SGT1Skp1 pathway). Further analysis to elucidate cell cycle components that can be assisted by TgHsp90 should be performed.

\section{STAGE DIFFERENTIATION}

Toxoplasma gondii interconversion between rapidly growing tachyzoites and latent encysted bradyzoites involves coordinated, sequential, morphological and metabolic processes accompanied by gene expression events (Dzierszinski et al. 2004). The TgHsp90 PPI network highlights the putative role of TgHsp90 in assisting glycolytic/respiration metabolism to obtain ATP, protein synthesis (which includes aminoacyl tRNA synthases), maturation of rRNAs, initiation and elongation factors and ribosomal proteins as well as protein degradation or processing (Table S1). These findings, obtained from $T$. gondii studies, have been further documented in other organisms (McClellan et al. 2007; Pavithra et al. 2007; Echeverria et al. 2011). In addition, the PPI network of $T$. gondii p23 in tachyzoites and bradyzoites has also allowed the retrieval of these types of proteins as putative interactors (Echeverria et al. 2010). Noteworthy, in the bradyzoite stage, the parasite mitochondrion is down-regulated, whereas in the highly replicative tachyzoite, oxidative phosphorylation is essential for sufficient ATP generation (Bohne et al. 1994; Tomavo and Boothroyd, 1995). Toxoplasma gondii lactate dehydrogenase LDH1 is present in tachyzoites while LDH2 is found in bradyzoites, and the over-expression of LDHs in tachyzoites has been shown to enhance the bradyzoite conversion during stress (Liwak and Ananvoranich, 2009). For this reason, it is reasonable to find out that several enzymes associated to ATP generation would be regulated by $\mathrm{TgHsp} 90$ under differentiation conditions, a situation that generates stress in the tachyzoite.

Another important change during parasite differentiation is the protein repertoire in which some proteins should be replaced for another set of new proteins. In this regard, during bradyzoite development, the $T$. gondii eukaryotic initiation factor 2 alpha subunit $(\mathrm{eIF} 2 \alpha)$ is phosphorylated, a PTM that restrains global protein synthesis, which persists in the bradyzoite stage (Narasimhan et al. 2008). Moreover, the inhibition of eIF $2 \alpha$ dephosphorylation by salubrinal and guanabenz has been shown to reduce tachyzoite replication and induce bradyzoite formation, blocking its reactivation (Konrad et al. 2013). The TgHsp90 PPI network includes 
elongation factors and several ribosomal proteins that are involved in protein biosynthesis as putative interactors, but not the eIF $2 \alpha$ kinase and $\operatorname{eIF} 2 \alpha$ (Table S1). Even though these data link TgHsp90 with assisting the protein biosynthesis pathway, it remains to be seen if TgHsp90 could be regulating this key metabolic process during parasite differentiation.

One of the most interesting and curious aspects concerning TgHsp90 and parasite differentiation relies on protein translocation to the nucleus (Echeverria et al. 2005). This could implicate a role in modulating transcription factors, chromatin and/ or RNA processing factors. Although the Hsp90 PPI network presents some components of chromatin and transcriptional machinery as putative TgHsp90 interactors (Table S1), there is an important gap in this field since only a basal transcriptional machinery has been detected in apicomplexan parasites (Meissner and Soldati, 2005). In addition, the involvement of an epigenetic mechanism in $T$. gondii gene regulation has been recently documented (Dalmasso et al. 2011; Croken et al. 2012). On the other hand, the role of Hsp90 in transcriptional activities, chromatin modulation, RNA processing/ splicing proteins and DNA replication/damageresponse has been recently reviewed (Sawarkar et al. 2012). Of note, a chromatin immunoprecipitation (ChIP) genome-wide analysis in Drosophila has shown that Hsp90 accumulates close to the transcription start sites of several protein-coding and miRNA-coding genes which are in a paused state of RNA polymerase II (Sawarkar et al. 2012). Further studies should be carried out to determine the importance of nuclear translocation of TgHsp90 during bradyzoite development. These studies should consider not only a putative role in assisting nuclear proteins but also a role in gene expression and/or maintaining the paused state of RNA pol II.

HOST-CELL INVASION

Toxoplasma gondii presents a compartmental organization similar to other eukaryotes and a group of apicomplexan specific-secretory organelles (micronemes and rhoptries) located at the apical tip of the parasite, which are involved in attachment and invasion (Carruthers and Boothroyd, 2007; Sharma and Chitnis, 2013). The contents of micronemes and rhoptries are discharged at the time of invasion. Another important organelle, the inner membrane complex (IMC), together with the plasma membrane (PM), forms the pellicle, a triple lipid bilayer typical of apicomplexan parasites. The IMC is of critical importance for the parasite's cellular processes such as replication, gliding motility and invasion (Lourido et al. 2012). Ahn et al. (2003) have shown that inhibition of $\mathrm{TgHsp} 90$ blocks parasite invasion into the host cell. These authors have also shown that TgHsp90 is secreted during invasion and antibodies against this chaperone also block the host cell entry of the parasite. In addition to secreted Hsp90, the PPI network retrieved some proteins associated with cell adhesion and parasite invasion such as micronemes and rhoptry proteins (Table S1). Maybe, intracellular Hsp90 also participates in regulating the secretion and/or maturation of these proteins.

\section{CONCLUSIONS}

As mentioned, toxoplasmosis is a disease of medical relevance mainly due to the possibility of reactivation of latent infections and vertical transmission. Thus, the absence of efficient treatment for chronic conditions makes this issue an interesting field of study. Because of this, the identification and characterization of essential or specific proteins which may be participating in essential cellular processes in $T$. gondii pathogenesis would allow the generation of novel therapies. The parasite chaperone Hsp90 is an interesting drug target against acute and latent toxoplasmosis (Angel et al. 2013).

Experimental data and database mining have allowed the identification of at least two of the Hsp90 cycles in T. gondii: the Hsp70/Hsp90 cycle or Hsp90-heterocomplex for transcription factors/ protein kinases and the cycle that involves SGT1 co-chaperones associated with the kinetochore assembly pathway. Both confirmed the involvement of TgHsp90 in cell cycle and development control, making this an interesting field of future studies. In addition, different putative Hsp90 interactors associated with key metabolic processes associated to differentiation, DNA replication, gene expression at transcriptional and post-transcriptional levels, have been detected. Hsp90 also has been found to be associated with $T$. gondii invasion. It is intriguing whether this role is fulfilled by the extracellular parasite Hsp90 and/or if TgHsp90 participates by assisting the function or secretion of some proteins belonging to the invasion pathways, such as rhoptries, micronemes, etc. Confirming some of these proteins as TgHsp90 interactors is one of our future goals to dissect the role of parasite Hsp90 in these processes.

Hsp90 is regulated by several PTMs such as phosphorylation, acetylation and S-nitrosylation. These PTMs are involved in modulating Hsp90 affinity for client proteins and co-chaperones. Although numerous putative phosphorylated sites have been found in $\mathrm{TgHsp} 90$, there are several phosphorylated sites in yeast and human Hsp90s with important regulatory functions, which are present in TgHsp90 but not in the phosphoproteome. Hsp90 is acetylated by HDAC enzymes. This PTM is highly interesting because it regulates the Hsp90 function and can be addressed by drugs that inhibit HDAC 
activity. Some but not all HDAC (e.g. HDAC6) have been identified as Hsp90 modifiers in the $T$. gondii database. We could speculate that the study of Hsp PTMs will bring up interesting findings in the future and promote novel anti-Hsp90 drugs.

In conclusion, $\mathrm{TgHsp} 90$ is a chaperone that may be modulating essential biological processes. Since studies on TgHsp90 are at an early stage, it is a promising field of study both to gain knowledge on the basic biology of the parasite and to evaluate it as a therapy target against toxoplasmosis.

\section{SUPPLEMENTARY MATERIAL}

To view supplementary material for this article, please visit http://dx.doi.org/10.1017/S0031182014000055.

\section{ACKNOWLEDGEMENTS}

SO Angel (Researcher) and L. Alomar (Postdoctoral Fellow) are members of the National Research Council of Argentina (CONICET). MJ Figueras is a Postdoctoral Fellow (NIAID-NIH grant). The authors wish to thank the toxoDB (www.toxodb.org).

\section{FINANCIAL SUPPORT}

This work was supported by National Institute of Health-National Institute of Allergy and Infectious Diseases (USA) 1R01AI083162-01 (to SOA) and by ANPCyT PICT 0623 (to SOA). Funding for the mass spectrometry analysis was provided by the Vermont Genetics Network/NIH Grant 8P20GM103449 from the INBRE programme of the NIGMS.

\section{REFERENCES}

Ahn, H. J., Kim, S. and Nam, W. (2003). Molecular cloning of the 82-kDa heat shock protein (HSP90) of Toxoplasma gondii associated with the entry into and growth in host cells. Biochemical and Biophysical Research Communication 311, 654-659.

Angel, S. O., Matrajt, M. and Echeverria, P. C. (2013). A review of recent patents on the protozoan parasite HSP90 as a drug target. Recent Patent on Biotechnology 7, 2-8.

Bedin, M., Catelli, M. G., Cabanie, L., Gaben, A. M. and Mester, J. (2009). Indirect participation of Hsp90 in the regulation of the cyclin $\mathrm{E}$ turnover. Biochemical Pharmacology 77, 151-158.

Behnke, M. S., Wootton, J. C., Lehmann, M. M., Radke, J. B., Lucas, O., Nawas, J., Sibley, L.D. and White, M.W. (2010). Coordinated progression through two subtranscriptomes underlies the tachyzoite cycle of Toxoplasma gondii. PLoS One 5, e12354.

Bogumil, D. and Dagan, T. (2012). Cumulative impact of chaperonemediated folding on genome evolution. Biochemistry 51, 9941-9953.

Bohne, W., Heesemann, J. and Gross, U. (1994). Reduced replication of Toxoplasma gondii is necessary for induction of bradyzoite-specific antigens: a possible role for nitric oxide in triggering stage conversion. Infection and Immunity 62, 1761-1767.

Burrows, F., Zhang, H. and Kamal, A. (2004). Hsp90 activation and cell cycle regulation. Cell Cycle 3, 1530-1536.

Carlier, Y., Truyens, C., Deloron, P. and Peyron, F. (2012). Congenital parasitic infections: a review. Acta Tropica 121, 55-70.

Carruthers, V. and Boothroyd, J.C. (2007). Pulling together: an integrated model of Toxoplasma cell invasion. Current Opinion in Microbiology 10, 83-89.

Catlett, M. G. and Kaplan, K. B. (2006). Sgt1p is a unique co-chaperone that acts as a client adaptor to link Hsp90 to Skp1p. Fournal of Biological Chemistry 281, 33739-33748.
Croken, M. M., Nardelli, S. C. and Kim, K. (2012). Chromatin modifications, epigenetics, and how protozoan parasites regulate their lives. Trends in Parasitology 28, 202-213.

Dalmasso, M. C., Jr., Sullivan, W. J. and Angel, S. O. (2011). Canonical and variant histones of protozoan parasites. Frontiers in Bioscience (Landmark Ed) 16, 2086-2105.

Dubey, J. P., Miller, N. L. and Frenkel, J. K. (1970). Toxoplasma gondii life cycle in cats. Fournal of the American Veterinary Medical Association 157, 1767-1770.

Dubey, J. P., Lindsay, D. S. and Speer, C. A. (1998). Structures of Toxoplasma gondii tachyzoites, bradyzoites, and sporozoites and biology and development of tissue cysts. Clinical Microbiology Reviews 11, 267-299.

Dzierszinski, F., Nishi, M., Ouko, L. and Roos, D. S. (2004). Dynamics of Toxoplasma gondii differentiation. Eukaryotic Cell 3, 992-1003.

Echeverria, P. C., Matrajt, M., Harb, O.S., Zappia, M.P., Costas, M. A., Roos, D. S., Dubremetz, J. F. and Angel, S. O. (2005) Toxoplasma gondii Hsp90 is a potential drug target whose expression and subcellular localization are developmentally regulated. Fournal of Molecular Biology 350, 723-734.

Echeverria, P.C., Figueras, M.J., Vogler, M., Kriehuber, T., de Miguel, N., Deng, B., Dalmasso, M.C., Matthews, D.E., Matrajt, M., Haslbeck, M., Buchner, J. and Angel, S. O. (2010). The Hsp90 co-chaperone p23 of Toxoplasma gondii: identification, functional analysis and dynamic interactome determination. Molecular and Biochemical Parasitology 172, 129-140.

Echeverria, P. C., Bernthaler, A., Dupuis, P., Mayer, B. and Picard, D. (2011). An interaction network predicted from public data as a discovery tool: application to the Hsp90 molecular chaperone machine. PLoS One 6, e26044.

Figueras, M. J., Martin, O. A., Echeverria, P.C., de Miguel, N., Naguleswaran, A., Jr., Sullivan, W. J., Corvi, M. M. and Angel, S. O. (2012). Toxoplasma gondii Sis1-like J-domain protein is a cytosolic chaperone associated to HSP90/HSP70 complex. International fournal for Biological Macromolecules 50, 725-733.

Gubbels, M. J., White, M. and Szatanek, T. (2008). The cell cycle and Toxoplasma gondii cell division: tightly knit or loosely stitched? International Fournal of Parasitology 38, 1343-1358.

Haarberg, H. E., Paraiso, K. H., Wood, E., Rebecca, V.W., Sondak, V. K., Koomen, J. M. and Smalley, K. S. (2013). Inhibition of Wee1, AKT, and CDK4 underlies the efficacy of the HSP90 inhibitor XL888 in an in vivo model of NRAS-mutant melanoma. Molecular Cancer Therapeutics 12, 901-912.

Halonen, S. K. and Weiss, L. M. (2013). Toxoplasmosis. Handbook of Clinical Neurology 114, 125-145.

Jackson, S. E. (2013). Hsp90: structure and function. Topics in Current Chemistry 328, 155-240.

Jeffers, V. and Jr., Sullivan, W. J. (2012). Lysine acetylation is widespread on proteins of diverse function and localization in the protozoan parasite Toxoplasma gondii. Eukaryotic Cell 11, 735-742.

Konrad, C., Queener, S. F., Wek, R. C. and Jr., Sullivan, W. J. (2013). Inhibitors of eIF2alpha dephosphorylation slow replication and stabilize latency in Toxoplasma gondii. Antimicrobial Agents and Chemotherapy 57, 1815-1822.

Kovacs, J. J., Murphy, P. J., Gaillard, S., Zhao, X., Wu, J. T., Nicchitta, C. V., Yoshida, M., Toft, D. O., Pratt, W. B. and Yao, T. P. (2005). HDAC6 regulates Hsp90 acetylation and chaperone-dependent activation of glucocorticoid receptor. Molecular Cell 18, 601-607.

Li, J. and Buchner, J. (2013). Structure, function and regulation of the hsp90 machinery. Biomedical fournal 36, 106-117.

Li, J., Soroka, J. and Buchner, J. (2012). The Hsp90 chaperone machinery: conformational dynamics and regulation by co-chaperones. Biochimica et Biophysica Acta 1823, 624-635.

Liwak, U. and Ananvoranich, S. (2009). Toxoplasma gondii: overexpression of lactate dehydrogenase enhances differentiation under alkaline conditions. Experimental Parasitology 122, 155-161.

Lourido, S., Tang, K. and Sibley, L.D. (2012). Distinct signalling pathways control Toxoplasma egress and host-cell invasion. EMBO Fournal 31, 4524-4534.

Makhnevych, T. and Houry, W. A. (2012). The role of Hsp90 in protein complex assembly. Biochimica et Biophysica Acta 1823, 674-682.

Martinez-Ruiz, A., Villanueva, L., Gonzalez de Orduna, C., Lopez-Ferrer, D., Higueras, M. A., Tarin, C., Rodriguez-Crespo, I., Vazquez, J. and Lamas, S. (2005). S-nitrosylation of Hsp90 promotes the inhibition of its ATPase and endothelial nitric oxide synthase regulatory activities. Proceedings of the National Academy of Sciences USA 102, 85258530 .

McClellan, A. J., Xia, Y., Deutschbauer, A. M., Davis, R.W., Gerstein, M. and Frydman, J. (2007). Diverse cellular functions of 
the Hsp90 molecular chaperone uncovered using systems approaches. Cell 131, 121-135.

Meissner, M. and Soldati, D. (2005). The transcription machinery and the molecular toolbox to control gene expression in Toxoplasma gondii and other protozoan parasites. Microbes and Infection 7, 1376-1384.

Miman, O., Mutlu, E.A., Ozcan, O., Atambay, M., Karlidag, R. and Unal, S. (2010). Is there any role of Toxoplasma gondii in the etiology of obsessive-compulsive disorder? Psychiatry Research 177, 263-265.

Mimnaugh, E. G., Worland, P. J., Whitesell, L. and Neckers, L. M (1995). Possible role for serine/threonine phosphorylation in the regulation of the heteroprotein complex between the hsp 90 stress protein and the pp60v-src tyrosine kinase. Fournal of Biological Chemistry 270 , 28654-28659

Mollapour, M. and Neckers, L. (2012). Post-translational modifications of Hsp90 and their contributions to chaperone regulation. Biochimica et Biophysica Acta 1823, 648-655.

Mollapour, M., Tsutsumi, S., Donnelly, A. C., Beebe, K., Tokita, M. J. Lee, M. J., Lee, S., Morra, G., Bourboulia, D., Scroggins, B. T., Colombo, G., Blagg, B.S., Panaretou, B., Stetler-Stevenson, W. G., Trepel, J. B., Piper, P. W., Prodromou, C., Pearl, L. H. and Neckers, L. (2010a). Swe1Wee1-dependent tyrosine phosphorylation of Hsp90 regulates distinct facets of chaperone function. Molecular Cell 37, 333-343.

Mollapour, M., Tsutsumi, S. and Neckers, L. (2010b). Hsp90 phosphorylation, Wee1 and the cell cycle. Cell Cycle 9, 2310-2316. Mollapour, M., Tsutsumi, S., Kim, Y.S., Trepel, J. and Neckers, L. (2011a). Casein kinase 2 phosphorylation of Hsp90 threonine 22 modulates chaperone function and drug sensitivity. Oncotarget 2, 407-417.

Mollapour, M., Tsutsumi, S., Truman, A. W., Xu, W., Vaughan, C. K., Beebe, K., Konstantinova, A., Vourganti, S., Panaretou, B. Piper, P.W., Trepel, J.B., Prodromou, C., Pearl, L.H. and Neckers, L. (2011b). Threonine 22 phosphorylation attenuates Hsp90 interaction with cochaperones and affects its chaperone activity. Molecular Cell 41, 672-681.

Murphy, P. J., Morishima, Y., Kovacs, J. J., Yao, T. P. and Pratt, W. B. (2005). Regulation of the dynamics of hsp90 action on the glucocorticoid receptor by acetylation/deacetylation of the chaperone. Fournal of Biological Chemistry 280, 33792-33799.

Narasimhan, J., Joyce, B. R., Naguleswaran, A., Smith, A. T., Livingston, M.R., Dixon, S.E., Coppens, I., Wek, R.C. and Jr., Sullivan, W. J. (2008). Translation regulation by eukaryotic initiation factor-2 kinases in the development of latent cysts in Toxoplasma gondii. Fournal of Biological Chemistry 283, 16591-16601.

Nathan, D. F. and Lindquist, S. (1995). Mutational analysis of Hsp90 function: interactions with a steroid receptor and a protein kinase. Molecular Cellular Biology 15, 3917-3925.

Ogiso, H., Kagi, N., Matsumoto, E., Nishimoto, M., Arai, R., Shirouzu, M., Mimura, J., Fujii-Kuriyama, Y. and Yokoyama, S. (2004). Phosphorylation analysis of $90 \mathrm{kDa}$ heat shock protein within the cytosolic arylhydrocarbon receptor complex. Biochemistry 43, 1551015519.

Park, J.H., Kim, S. H., Choi, M. C., Lee, J., Oh, D. Y., Im, S. A., Bang, Y. J. and Kim, T. Y. (2008). Class II histone deacetylases play pivotal roles in heat shock protein 90-mediated proteasomal degradation of vascular endothelial growth factor receptors. Biochemical and Biophysical Research Communication 368, 318-322.

Park, M. H., Kwon, Y.J., Jeong, H.Y., Lee, H. Y., Hwangbo, Y., Yoon, H. J. and Shim, S. H. (2012). Association between intracellular infectious agents and schizophrenia. Clinical Psychopharmacology and Neuroscience 10, 117-123.

Pavithra, S. R., Kumar, R. and Tatu, U. (2007). Systems analysis of chaperone networks in the malarial parasite Plasmodium falciparum. PLOS Computational Biology 3, 1701-1715.

Pearl, L. H., Prodromou, C. and Workman, P. (2008). The Hsp90 molecular chaperone: an open and shut case for treatment. Biochemical Fournal 410, 439-453.

Picard, D. (2002). Heat-shock protein 90, a chaperone for folding and regulation. Cellular and Molecular Life Sciences 59, 1640-1648.

Picard, D., Suslova, E. and Briand, P. A. (2006). 2-color photobleaching experiments reveal distinct intracellular dynamics of two components of the Hsp90 complex. Experimental Cell Research 312, 3949-3958.

Pratt, W. B. and Toft, D. O. (2003). Regulation of signaling protein function and trafficking by the hsp $90 /$ hsp 70 -based chaperone machinery. Experimental Biology and Medicine 228, 111-133.

Prodromou, C. and Pearl, L.H. (2003). Structure and functional relationships of Hsp90. Current Cancer Drug Targets 3, 301-323.

Prodromou, C., Roe, S. M., O’Brien, R., Ladbury, J. E., Piper, P. W. and Pearl, L.H. (1997). Identification and structural characterization of the ATP/ADP-binding site in the Hsp90 molecular chaperone. Cell 90, $65-75$.

Radke, J. R., Striepen, B., Guerini, M. N., Jerome, M. E., Roos, D. S. and White, M.W. (2001). Defining the cell cycle for the tachyzoite stage of Toxoplasma gondii. Molecular and Biochemical Parasitology 115, 165-175. Radke, J. R., Guerini, M. N., Jerome, M. and White, M. W. (2003). A change in the premitotic period of the cell cycle is associated with bradyzoite differentiation in Toxoplasma gondii. Molecular and Biochemical Parasitology 131, 119-127

Retzlaff, M., Hagn, F., Mitschke, L., Hessling, M., Gugel, F., Kessler, H., Richter, K. and Buchner, J. (2010). Asymmetric activation of the hsp90 dimer by its cochaperone aha1. Molecular Cell 37, 344-354. Rochani, A. K., Singh, M. and Tatu, U. (2013). Heat shock protein 90 inhibitors as broad spectrum anti-infectives. Current Pharmaceutical Design 19, 377-386.

Roy, N., Nageshan, R. K., Ranade, S. and Tatu, U. (2012). Heat shock protein 90 from neglected protozoan parasites. Biochimica et Biophysica Acta 1823, 707-711.

Sawarkar, R., Sievers, C. and Paro, R. (2012). Hsp90 globally targets paused RNA polymerase to regulate gene expression in response to environmental stimuli. Cell 149, 807-818.

Scroggins, B. T., Robzyk, K., Wang, D., Marcu, M. G., Tsutsumi, S. Beebe, K., Cotter, R. J., Felts, S., Toft, D., Karnitz, L., Rosen, N. and Neckers, L. (2007). An acetylation site in the middle domain of Hsp90 regulates chaperone function. Molecular Cell 25, 151-159.

Shao, J., Hartson, S. D. and Matts, R. L. (2002). Evidence that protein phosphatase 5 functions to negatively modulate the maturation of the Hsp90-dependent heme-regulated eIF2alpha kinase. Biochemistry 41, 6770-6779.

Sharma, P. and Chitnis, C. E. (2013). Key molecular events during host cell invasion by Apicomplexan pathogens. Current Opinion in Microbiology 16, 432-437.

Shonhai, A., Maier, A. G., Przyborski, J. M. and Blatch, G. L. (2011). Intracellular protozoan parasites of humans: the role of molecular chaperones in development and pathogenesis. Protein and Peptide Letters 18, 143-157.

Soroka, J., Wandinger, S. K., Mausbacher, N., Schreiber, T., Richter, K., Daub, H. and Buchner, J. (2012). Conformational switching of the molecular chaperone Hsp90 via regulated phosphorylation. Molecular Cell 45, 517-528.

Stebbins, C. E., Russo, A. A., Schneider, C., Rosen, N., Hartl, F. U. and Pavletich, N.P. (1997). Crystal structure of an Hsp90-geldanamycin complex: targeting of a protein chaperone by an antitumor agent. Cell $\mathbf{8 9}$, 239-250.

Striepen, B., Jordan, C. N., Reiff, S. and van Dooren, G. G. (2007) Building the perfect parasite: cell division in apicomplexa. PLoS Pathogen $\mathbf{3}$, e78.

Suvorova, E. S., Radke, J. B., Ting, L. M., Vinayak, S., Alvarez, C. A., Kratzer, S., Kim, K., Striepen, B. and White, M. W. (2013). A nucleolar AAA-NTPase is required for parasite division. Molecular Microbiology $\mathbf{9 0}$, 338-355. doi: $10.1111 / \mathrm{mmi} .12367$.

Tenter, A. M., Heckeroth, A. R. and Weiss, L. M. (2000). Toxoplasma gondii: from animals to humans. International Fournal for Parasitology $\mathbf{3 0}$, $1217-1258$

Tomavo, S. and Boothroyd, J. C. (1995). Interconnection between organellar functions, development and drug resistance in the protozoan parasite, Toxoplasma gondii. International Fournal for Parasitology 25, 1293-1299.

Treeck, M., Sanders, J. L., Elias, J. E. and Boothroyd, J. C. (2011). The phosphoproteomes of Plasmodium falciparum and Toxoplasma gondii reveal unusual adaptations within and beyond the parasites' boundaries. Cell Host and Microbe 10, 410-419.

Tsutsumi, S., Mollapour, M., Graf, C., Lee, C. T., Scroggins, B. T., Xu, W., Haslerova, L., Hessling, M., Konstantinova, A. A., Trepel, J. B., Panaretou, B., Buchner, J., Mayer, M.P., Prodromou, C. and Neckers, L. (2009). Hsp90 charged-linker truncation reverses the functional consequences of weakened hydrophobic contacts in the N domain. Nature Structural and Molecular Biology 16, 1141-1147.

Ueno, A., Dautu, G., Haga, K., Munyaka, B., Carmen, G., Kobayashi, Y. and Igarashi, M. (2011). Toxoplasma gondii: a bradyzoite-specific DnaK-tetratricopeptide repeat (DnaK-TPR) protein interacts with p23 co-chaperone protein. Experimental Parasitology 127, 795-803.

Vanagas, L., Jeffers, V., Bogado, S. S., Dalmasso, M. C., Jr., Sullivan, W. J. and Angel, S. O. (2012). Toxoplasma histone acetylation remodelers as novel drug targets. Expert Review of Anti-Infective Therapy 10, 1189-1201. 
Wang, X., Song, X., Zhuo, W., Fu, Y., Shi, H., Liang, Y., Tong, M., Chang, G. and Luo, Y. (2009). The regulatory mechanism of Hsp90alpha secretion and its function in tumor malignancy. Proceedings of the National Academy of Sciences USA 106, 21288-21293.

Xu, W., Mollapour, M., Prodromou, C., Wang, S., Scroggins, B. T., Palchick, Z., Beebe, K., Siderius, M., Lee, M. J., Couvillon, A., Trepel, J. B., Miyata, Y., Matts, R. and Neckers, L. (2012). Dynamic tyrosine phosphorylation modulates cycling of the HSP90-P50(CDC37)AHA1 chaperone machine. Molecular Cell 47, 434-443.

Xue, B., Jeffers, V., Sullivan, W. J. and Uversky, V. N. (2013). Protein intrinsic disorder in the acetylome of intracellular and extracellular Toxoplasma gondii. Molecular BioSystems 9, 645-657.
Yu, X., Guo, Z.S., Marcu, M. G., Neckers, L., Nguyen, D. M., Chen, G. A. and Schrump, D. S. (2002). Modulation of p53, ErbB1, ErbB2, and Raf-1 expression in lung cancer cells by depsipeptide FR901228. Fournal of the National Cancer Institute 94, 504-513.

Zhou, Q., Agoston, A. T., Atadja, P., Nelson, W. G. and Davidson, N. E. (2008). Inhibition of histone deacetylases promotes ubiquitin-dependent proteasomal degradation of DNA methyltransferase 1 in human breast cancer cells. Molecular Cancer Research 6, 873-883.

Zhou, W., Quan, J. H., Lee, Y.H., Shin, D. W. and Cha, G. H. (2013).

Proliferation require down-regulation of host Nox 4 expression via activation of PI3 Kinase/Akt signaling pathway. PLoS ONE 8, e66306. 